\title{
Greedy method for robust linear regression
}

\author{
Tao Sun ${ }^{\mathrm{a}, *}$, Lizhi Cheng ${ }^{\mathrm{a}, \mathrm{c}}$, Hao Jiang ${ }^{\mathrm{b}}$ \\ ${ }^{a}$ College of Science, National University of Defense Technology, Changsha, Hunan, \\ China, 410073. \\ ${ }^{b}$ College of Computer, National University of Defense Technology, Hunan, Changsha, \\ China, 410073. \\ ${ }^{c}$ The State Key Laboratory for High Performance Computation, National University of \\ Defense Technology, Hunan, Changsha, China, 410073.
}

\begin{abstract}
In this paper, we propose a greedy method to estimate the unknown vector from linear observation with sparse noise. We prove that the algorithm can reconstruct the vector provided the sampling matrix satisfies certain condition and the noise is sparse. We also prove that such a condition holds with high probability for random matrix if its scale satisfies certain assumption. Numerical results are provided to demonstrate the efficiency of the algorith$\mathrm{m}$. And we also consider using the algorithm for salt\&pepper noise removal in signal processing.
\end{abstract}

Keywords: Greedy method; robust linear regression; sparse noise

\section{Introduction}

Linear regression is old but important problem which finds interests in data analysis, signal processing and statistics [1,2]. Given a pair sequence $\left\{\left(\mathbf{A}_{i}, b_{i}\right)\right\}_{i=1,2, \ldots, M}$, it is routinely assumed that

$$
b_{i}=\left\langle\mathbf{A}_{i}, \mathbf{x}\right\rangle+e_{i}^{*},
$$

where $\mathbf{A}_{i} \in \mathbb{R}^{N}$, and $b_{i} \in \mathbb{R}$, and $e_{i}^{*} \in \mathbb{R}$ is the noise, and $\mathbf{x} \in \mathbb{R}^{N}$ is unknown vector. In information theory, procedure (1) is also called as sampling. Original linear regression always assumes that $\mathbf{e} \in \mathbb{R}^{N}$ is a white Gaussian

\footnotetext{
${ }^{*}$ Corresponding author

Email addresses: nudtsuntao@163.com,nudttaosun@gmail.com (Tao Sun), clzcheng@nudt. edu.cn (Lizhi Cheng), haojiang@nudt.edu.cn (Hao Jiang)
} 
noise, and aim to estimate $\mathbf{x}$ by minimizing the sum of squared residuals $\sum_{i=1}^{M}\left(b_{i}-\mathbf{A}_{i} \mathbf{x}\right)^{2}$; in matrix form, the problem can be presented as

$$
\min _{\mathbf{x}}\|\mathbf{b}-\mathbf{A} \mathbf{x}\|_{2}
$$

where $\mathbf{A}=\left[\mathbf{A}_{1}, \mathbf{A}_{2}, \ldots, \mathbf{A}_{M}\right]^{\top}$ and $\mathbf{b}=\left[b_{1}, b_{2}, \ldots, b_{M}\right]^{\top}$. However, in applications, the noise may be of other form, like sparsity (salt\&pepper noise). As a result, quite various robust linear regressions are developed [3, 4].

The $M$-Estimates method [5] for the robust linear regression is an effective tool. In fact, this is a wide class of methods depending on an auxiliary function $\varphi$. If $\varphi(t)=|t|$, the method reduces to the well-known Least Absolute Deviations (LAD) [7]. In other literatures, it is also called as median regression [6]. Mathematically, the LAD problem can be formulated as

$$
\min _{\mathbf{x} \in \mathbb{R}^{N}}\|\mathbf{b}-\mathbf{A} \mathbf{x}\|_{1}
$$

The $\ell_{1}$ norm is routinely used to measure the sparsity of a vector in information theory and signal processing. From such a perspective, model (3) can naturally obtain a vector such that the residual (noise part) is sparse. The LAD is also known as $\ell_{1}$ regression problem which is geometry interpreted as fitting a hyperplane for a given set with $m$ points $[8,9]$. It is well known that the LAD is an instance of linear programming. Due to that $\ell_{1}$ norm is indifferentiable, most direct algorithms perform slowly. In statics, it usually holds that $m \gg n$ and $\operatorname{rank}(\mathbf{A})=n$. Under the $\ell_{1}$ conditioned assumption $\|\mathbf{x}\|_{1} / n \sqrt{n} \leq\|\mathbf{A x}\|_{1} \leq\|\mathbf{x}\|_{1}$, paper[10] proposes the subgradient descent and the sampling method to solve the LAD approximately. In latter papers, the sampling method receives much more attentions in that $m \gg n[12,11]$. The sampling method can be regarded as a precondition method, and at last we still need to solve a LAD problem. Due to that M-estimators are not robust to high-leverage outliers, the Least Median of Squares (LMS) estimator[24] is proposed as

$$
\min _{x} \operatorname{med}(\mathbf{A x}-\mathbf{b})
$$

where med represents the median of a vector. Other methods like the MMestimates and S-estimate $[26,27]$ are also proposed. These two methods consist of several steps and are much more complicated. More details can be founded in $[26,27]$. 
Apart from the statical problems, robust estimates also have applications in imaging science. The TV-L $\mathrm{L}_{1}$ is right a typical example[13] which reads as

$$
\min _{\mathbf{x}}\|\mathbf{d}-\mathbf{x}\|_{1}+\lambda\|\mathbf{T} \mathbf{x}\|_{1}
$$

where $\mathbf{T}$ is the TV operator[14]. It is a linear operator; in one-dimension case, it is a matrix and $\mathbf{T} \mathbf{x}=\left[\mathbf{x}_{1}-\mathbf{x}_{2}, \mathbf{x}_{2}-\mathbf{x}_{3}, \ldots, \mathbf{x}_{N-1}-\mathbf{x}_{N}\right] \in \mathbb{R}^{N-1}$. Letting $\widehat{\mathbf{A}}:=[\lambda \mathbf{T}, \mathbf{I d}]^{\top}$ and $\widehat{\mathbf{b}}=[\mathbf{0}, \mathbf{d}]$, then (5) turns to

$$
\min _{\mathbf{x}}\|\widehat{\mathbf{b}}-\widehat{\mathbf{A}} \mathbf{x}\|_{1} \text {. }
$$

In this paper, we focus on the case that the noise is sparse and present a greedy method to estimate the original vector. We highlight that we do not use a greedy method to solve the LAD or other models but estimate $\mathbf{x}$ for (1) directly when the noise is sparse. Such a greedy method is quite faster than existing robust methods. We provide a theorem about the performance of this algorithm under several assumptions. Numerical results demonstrate the efficiency of this algorithm. From the statements previously, we can see that the $\mathrm{TV}-\mathrm{L}_{1}$ problem can be also regarded as estimating a vector for sparsely noised linear system. We also use the greedy method to reconstruct a one-dimensional signal.

The rest of the paper is organized as follows. In section 2, we describe the details of the algorithm. In section 3, we study the performance of the algorithm. In section 4, we provide several numerical results. Section 5 concludes the paper.

\section{Notations}

We adopt the notations described in this section throughout the paper. Let $\mathrm{x}$ be a vector and $x_{i}$ be its $i$-th component. The $l_{1}$ and $l_{2}$ norms of a vector $\mathbf{x}$ are denoted by $\|\mathbf{x}\|_{1}:=\sum\left|x_{i}\right|$ and $\|\mathbf{x}\|_{2}:=\left(\sum\left|x_{i}\right|\right)^{1 / 2}$, respectively. Let $A \in \mathbb{R}^{m \times n}$ be a matrix, and $A_{i} \in \mathcal{R}^{n}$ be $i$-th column of $\mathbf{A}$, and $\mathbf{A}_{(i)} \in \mathbb{R}^{m}$ be $i$-th row of $\mathbf{A}$. For a set $S=\left\{i_{1}, i_{2}, \ldots, i_{s}\right\} \subseteq\{1,2, \ldots, N\}, \mathbf{A}_{S}:=$ $\left\{\mathbf{A}_{i_{1}}, \mathbf{A}_{i_{2}}, \ldots, \mathbf{A}_{i_{s}}\right\} \in \mathbb{R}^{M \times s}$. The spectral norm of $\mathbf{A}$ is denoted by $\|\mathbf{A}\|_{2}:=$ $\max _{\|\mathbf{x}\|_{2}=1}\left\{\|\mathbf{A x}\|_{2}\right\}$. The minimum nonzero singular value of $\mathbf{A}$ is denoted by $\sigma_{\min }(\mathbf{A})$. Let $V$ be a convex set, the projection of vector $\mathbf{x}$ to $V$ is denoted as $\mathbf{P}_{V}(\mathbf{x}):=\arg \min _{\mathbf{y} \in V}\|\mathbf{x}-\mathbf{y}\|_{2}$. 


\section{The greedy methodology}

The algorithm proposed in this section is motivated by the famous Orthogonal Matching Pursuit (OMP) $[15,16,17]$. For a given pair $\left(\mathbf{x}^{0}, \mathbf{e}^{0}\right)$ and set $S^{0}$, in the $k$-th iteration, we first identify a coordinate $j$ by the following strategy

$$
j \in \arg \max _{i}\left|\mathbf{b}-\mathbf{A} \mathbf{x}^{k}-\mathbf{e}^{k}\right|_{i} .
$$

And then, we set $S^{k+1}=S^{k} \bigcup j$ and minimize the following problem

$$
\left(\mathbf{x}^{k+1}, \mathbf{e}^{k+1}\right) \in \arg \min _{\mathbf{x}, \operatorname{supp}(\mathbf{e}) \subseteq S^{k+1}}\|\mathbf{b}-\mathbf{A x}-\mathbf{e}\|_{2} .
$$

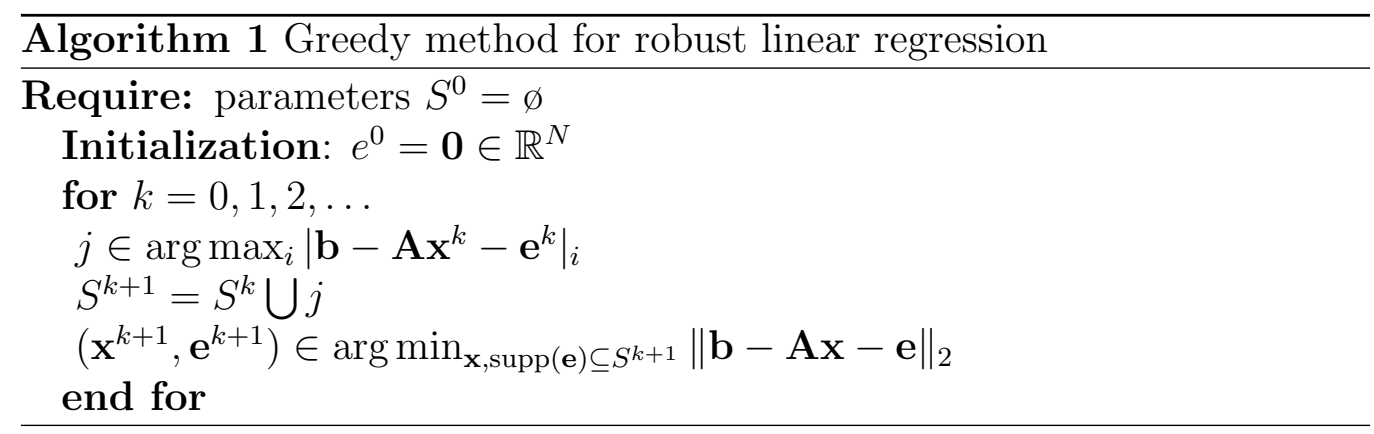

In each iteration of the algorithm, we need to solve a least square problem. That is the most costly part in the algorithm. If the matrix is not full columnrank, the problem has infinite solutions. In this case, we choose the one which has the least length. Then, letting $\chi^{k+1}$ be the solution set of problem (8), we choose $\mathbf{x}^{k+1}=\mathbf{P}_{\chi^{k+1}}(\mathbf{0})$, where $\mathbf{0} \in \mathbb{R}^{N}$. Note the convexity of $\chi^{k+1}, \mathbf{x}^{k+1}$ is unique. In fact, in this case, $[A 3,[18]]$ shows that

$$
\mathbf{x}^{k+1}=\left\{\left[\mathbf{A}, \mathbf{I d}_{S^{k+1}}\right]^{\dagger} \mathbf{b}\right\}_{\{1,2, \ldots, N\}},
$$

where $(\cdot)^{\dagger}$ denotes the pseudo-inverse [19] and Id is the identity matrix, i.e.,

$$
\mathbf{I d}=\left(\begin{array}{cccc}
1 & 0 & \cdots & 0 \\
0 & 1 & \ddots & \vdots \\
\vdots & \ddots & \ddots & 0 \\
0 & \cdots & 0 & 1
\end{array}\right)=\left[\mathbf{e}_{1}, \mathbf{e}_{2}, \ldots, \mathbf{e}_{N}\right]
$$


and $\mathbf{e}_{i}=[\underbrace{0, \ldots, 0}_{i-1}, 1,0, \ldots]^{\top}$.

The greedy strategy used here is similar to the one in OMP which aims to reduce the length of $\mathbf{b}-\mathbf{A} \mathbf{x}^{k}-\mathbf{e}^{k}$ as large as possible in each iteration. The stopping criterion is set as $\left\|\mathbf{b}-\mathbf{A} \mathbf{x}^{k}-\mathbf{e}^{k}\right\| \leq \eta$ for some small scale $\eta$. Such a rule is quite natural. Here, we provide a lemma to demonstrate the reasonableness of this strategy.

Lemma 2.1. Let $\boldsymbol{A} \in \mathbb{R}^{M \times N}$ be a matrix. Given a set $S \subseteq\{1,2, \ldots, M\}$ and $\boldsymbol{y}, \operatorname{supp}(\boldsymbol{u}) \subseteq S$, and $j \in\{1,2, \ldots, M\} \backslash S$. Let

$$
(\boldsymbol{z}, \boldsymbol{v}) \in \arg \min _{\boldsymbol{x}, \operatorname{supp}(\boldsymbol{e}) \subseteq S}\|\boldsymbol{b}-\boldsymbol{A} \boldsymbol{x}-\boldsymbol{e}\|_{2} .
$$

Then, it holds that

$$
\|\boldsymbol{b}-\boldsymbol{A} \boldsymbol{z}-\boldsymbol{v}\|_{2}^{2} \leq\|\boldsymbol{b}-\boldsymbol{A} \boldsymbol{y}-\boldsymbol{u}\|_{2}^{2}-\left|(\boldsymbol{b}-\boldsymbol{A} \boldsymbol{y}-\boldsymbol{u})_{j}\right|^{2} .
$$

Proof. Let $\varepsilon_{j}$ be the $j$-th element in the canonical basis of $\mathbb{R}^{N}$. Then, for any $t \in \mathbb{R}, \mathbf{u}+t \varepsilon_{j}$ is supported on $S \bigcup j$. That is also

$$
\begin{aligned}
\|\mathbf{b}-\mathbf{A z}-\mathbf{v}\|_{2}^{2} & \leq\left\|\mathbf{b}-\mathbf{A y}-\mathbf{u}-t \varepsilon_{j}\right\|_{2}^{2} \\
& =\|\mathbf{b}-\mathbf{A y}-\mathbf{u}\|_{2}^{2}-2 t\left\langle\mathbf{b}-\mathbf{A y}-\mathbf{u}, \varepsilon_{j}\right\rangle+t^{2} .
\end{aligned}
$$

Note that $\left\langle\mathbf{b}-\mathbf{A y}-\mathbf{u}, \varepsilon_{j}\right\rangle=(\mathbf{b}-\mathbf{A y}-\mathbf{u})_{j}$. Letting $t=(\mathbf{b}-\mathbf{A y}-\mathbf{u})_{j}$, we can immediately obtain the result.

\section{Performance analysis}

In this part, we analyze the performance of the algorithm. First, we collect several useful definitions and lemmas.

Definition 3.1. For a matrix A, we define

$$
\beta_{t}(\mathbf{A}):=\max _{\mathbf{x} \neq \mathbf{0}} \frac{\|\mathbf{A} \mathbf{x}\|_{2}}{\|\mathbf{x}\|_{2}},
$$

where $\sharp(\operatorname{supp}(\mathbf{x})) \leq t$.

Remark 3.2. The definition has deep relationship with the Restricted Isometry Property (RIP)[20]. If the matrix has $s$-order RIP, then,

$$
\beta_{s}(\mathbf{A}) \leq 1+\delta_{s}
$$

where $\delta_{s}$ is $s$-order RIP constant. 
Lemma 3.3. Let $\boldsymbol{x}$ and $\boldsymbol{e}$ be generated by (8), if $\sigma_{\min }(\boldsymbol{A})>0$, we can also obtain that

$$
\left\|\boldsymbol{e}-\boldsymbol{e}^{*}\right\|_{2} \geq \frac{\sigma_{\min }^{2}(\boldsymbol{A})\left\|\boldsymbol{x}-\boldsymbol{x}^{*}\right\|_{2}}{\|\boldsymbol{A}\|_{2}}
$$

Proof. The K.K.T condition of problem (8) is

$$
\mathbf{A}^{\top}(\mathbf{b}-\mathbf{A x}-\mathbf{e})=\mathbf{0} .
$$

Note that $\mathbf{b}=\mathbf{A} \mathbf{x}^{*}+\mathbf{e}^{*}$, then, we have that

$$
\mathbf{A}^{\top} \mathbf{A}\left(\mathbf{x}^{*}-\mathbf{x}\right)=\mathbf{A}^{\top}\left(\mathbf{e}-\mathbf{e}^{*}\right) \text {. }
$$

Hence, we have that

$$
\begin{aligned}
\|\mathbf{A}\|_{2}\left\|\mathbf{e}-\mathbf{e}^{*}\right\|_{2} & \geq\left\|\mathbf{A}^{\top}\left(\mathbf{e}-\mathbf{e}^{*}\right)\right\|_{2} \\
& =\left\|\mathbf{A}^{\top} \mathbf{A}\left(\mathbf{x}^{*}-\mathbf{x}\right)\right\|_{2} \\
& \geq \sigma_{\min }^{2}(\mathbf{A})\left\|\mathbf{x}^{*}-\mathbf{x}\right\|_{2}
\end{aligned}
$$

Define $\varepsilon_{k}$ to be the $\mathrm{k}$-th element in the canonical basis of $\mathbb{R}^{N}$. Without loss of generality, we assume that the support of $\mathbf{e}^{*}$ is $\{1,2, \ldots, s\}$. Given $\mathbf{y}$, $\mathbf{z}$ and $k$, denote that

$$
\phi_{k}(\mathbf{y}, \mathbf{z})=\left\langle\mathbf{A y}+\mathbf{z}, \varepsilon_{k}\right\rangle .
$$

And we denote that $\phi_{0}(\mathbf{y}, \mathbf{z})=\max _{1 \leq i \leq s}\left|\phi_{k}(\mathbf{y}, \mathbf{z})\right|$.

Lemma 3.4. Assume that $\|\boldsymbol{z}\|_{2} \neq 0$ and

$$
\sqrt{s+1} \beta_{s+1}\left(\boldsymbol{A}^{\top}\right)\|\boldsymbol{y}\|_{2}<\|\boldsymbol{z}\|_{2} \text {. }
$$

For any vector $\boldsymbol{z}$ satisfies that $\operatorname{supp}(\boldsymbol{z}) \subseteq\{1,2, \ldots, s\}$, we have that $\phi_{0}(\boldsymbol{y}, \boldsymbol{z})>$ $\phi_{k}(\boldsymbol{y}, \boldsymbol{z})$ if $k>s$.

Proof. We can easily have that

$$
\begin{aligned}
\langle\mathbf{A y}+\mathbf{z}, \mathbf{z}\rangle & =\sum_{i=1}^{s} z_{i}\left\langle\mathbf{A y}+\mathbf{z}, \varepsilon_{i}\right\rangle \\
& =\sum_{i=1}^{s} z_{i} \phi_{i}(\mathbf{y}, \mathbf{z}) \\
& \leq \sum_{i=1}^{s}\left|z_{i}\right| \phi_{0}(\mathbf{y}, \mathbf{z}) \\
& =\phi_{0}(\mathbf{y}, \mathbf{z})\|\mathbf{z}\|_{1} \\
& \leq \sqrt{s} \phi_{0}(\mathbf{y}, \mathbf{z})\|\mathbf{z}\|_{2} .
\end{aligned}
$$


Then, we obtain that

$$
\begin{aligned}
\sqrt{s}\left[\phi_{0}(\mathbf{y}, \mathbf{z})-\phi_{k}(\mathbf{y}, \mathbf{z})\right]\|\mathbf{z}\|_{2} & \geq\langle\mathbf{A y}+\mathbf{z}, \mathbf{z}\rangle-\sqrt{s} \phi_{k}(\mathbf{y}, \mathbf{z})\|\mathbf{z}\|_{2} \\
& =\langle\mathbf{A y}+\mathbf{z}, \mathbf{z}\rangle-\sqrt{s}\left\langle\mathbf{A y}+\mathbf{z}, \varepsilon_{k}\right\rangle\|\mathbf{z}\|_{2} .
\end{aligned}
$$

Note that $\operatorname{supp}(\mathbf{z}) \subseteq\{1,2, \ldots, s\}$, then, $\left\langle\mathbf{z}, \varepsilon_{k}\right\rangle=0$ if $k>s$. Then, (19) can be represented as

$$
\begin{aligned}
\langle\mathbf{A y}+\mathbf{z}, \mathbf{z}\rangle-\sqrt{s}\left\langle\mathbf{A y}+\mathbf{z}, \varepsilon_{k}\right\rangle\|\mathbf{z}\|_{2} & =\langle\mathbf{A y}, \mathbf{z}\rangle+\|\mathbf{z}\|_{2}^{2}-\sqrt{s}\left\langle\mathbf{A y}, \varepsilon_{k}\right\rangle\|\mathbf{z}\|_{2} \\
& =\left\langle\mathbf{A y}, \mathbf{z}-\sqrt{s}\|\mathbf{z}\|_{2} \varepsilon_{k}\right\rangle+\|\mathbf{z}\|_{2}^{2} \\
& =\left\langle\mathbf{y}, \mathbf{A}^{\top}\left(\mathbf{z}-\sqrt{s}\|\mathbf{z}\|_{2} \varepsilon_{k}\right)\right\rangle+\|\mathbf{z}\|_{2}^{2}(20)
\end{aligned}
$$

It is easy to see that $\mathbf{z}-\sqrt{s}\|\mathbf{z}\|_{2} \varepsilon_{k}$ is $s+1$-sparse and

$$
\|\mathbf{z}-\sqrt{s}\| \mathbf{z}\left\|_{2} \varepsilon_{k}\right\|_{2}=\sqrt{s+1}\|\mathbf{z}\|_{2} .
$$

Thus, we have

$$
\left|\left\langle\mathbf{y}, \mathbf{A}^{\top}\left(\mathbf{z}-\sqrt{s}\|\mathbf{z}\|_{2} \varepsilon_{k}\right)\right\rangle\right| \leq \sqrt{s+1} \beta_{s+1}\left(\mathbf{A}^{\top}\right)\|\mathbf{y}\|_{2}\|\mathbf{z}\|_{2} .
$$

That also means

$$
\begin{aligned}
\left\langle\mathbf{y}, \mathbf{A}^{\top}\left(\mathbf{z}-\sqrt{s}\|\mathbf{z}\|_{2} \varepsilon_{k}\right)\right\rangle & \geq-\sqrt{s+1} \beta_{s+1}\left(\mathbf{A}^{\top}\right)\|\mathbf{y}\|_{2}\|\mathbf{z}\|_{2} \\
& >-\|z\|_{2}^{2},
\end{aligned}
$$

where the second inequality depends on the assumption $\sqrt{s+1} \beta_{s+1}\left(\mathbf{A}^{\top}\right)\|\mathbf{y}\|_{2}<$ $\|\mathbf{z}\|_{2}$. Substituting (23) into (20), we then derive that

$$
\langle\mathbf{A y}+\mathbf{z}, \mathbf{z}\rangle-\sqrt{s}\left\langle\mathbf{A y}+\mathbf{z}, \varepsilon_{k}\right\rangle\|\mathbf{z}\|_{2}>0
$$

Combining (24) and (19), we then obtain that

$$
\phi_{0}(\mathbf{y}, \mathbf{z})>\phi_{k}(\mathbf{y}, \mathbf{z})
$$

if $k>s$.

Theorem 3.5. If

$$
\frac{\sigma_{\min }^{2}(\boldsymbol{A})}{\|\boldsymbol{A}\|_{2}}>\sqrt{s+1} \beta_{s+1}\left(\boldsymbol{A}^{\top}\right)
$$

then for any s-sparse noise $\boldsymbol{e}^{*}$, Algorithm 1 will recover $\boldsymbol{x}^{*}$ from $\boldsymbol{b}=\boldsymbol{A} \boldsymbol{x}+\boldsymbol{e}^{*}$ in $s$ iterations. 
Proof. In the $k$-th iteration of Algorithm 1, we can see

$$
\begin{aligned}
\left(\mathbf{b}-\mathbf{A} \mathbf{x}^{k}-\mathbf{e}^{k}\right)_{i} & =\left[\mathbf{A}\left(\mathbf{x}^{*}-\mathbf{x}^{k}\right)+\mathbf{e}^{*}-\mathbf{e}^{k}\right]_{i} \\
& =\phi_{i}\left(\mathbf{x}^{*}-\mathbf{x}^{k}, \mathbf{e}^{*}-\mathbf{e}^{k}\right) .
\end{aligned}
$$

In the first iteration, $\mathbf{x}^{0}=\mathbf{0}$, and $\mathbf{x}^{*}-\mathbf{x}^{0}=\mathbf{x}^{*} \neq \mathbf{0}$. From the definition $(17)$,

$$
\left|\phi_{i}\left(\mathbf{x}^{*}-\mathbf{x}^{k}, \mathbf{e}^{*}-\mathbf{e}^{k}\right)\right| \leq \phi_{0}\left(\mathbf{x}^{*}-\mathbf{x}^{k}, \mathbf{e}^{*}-\mathbf{e}^{k}\right), i \in\{1,2, \ldots, s\} .
$$

First we prove that the index $j$ selected in the first iteration must be located in $\{1,2, \ldots, s\}$. Otherwise, i.e., if $j>s$, we will have that

$$
\left|\phi_{j}\left(\mathbf{x}^{*}-\mathbf{x}^{k}, \mathbf{e}^{*}-\mathbf{e}^{k}\right)\right|<\phi_{0}\left(\mathbf{x}^{*}-\mathbf{x}^{k}, \mathbf{e}^{*}-\mathbf{e}^{k}\right) .
$$

from Lemmas 3.3 and 3.4. However, in the scheme of Algorithm 1,

$$
j \in \arg \max _{i}\left|\mathbf{b}-\mathbf{A} \mathbf{x}^{k}-\mathbf{e}^{k}\right|_{i}=\arg \max _{i}\left|\phi_{i}\left(\mathbf{x}^{*}-\mathbf{x}^{k}, \mathbf{e}^{*}-\mathbf{e}^{k}\right)\right|,
$$

which leads to a contraction to (28).

Similarly, in the second iteration, we also have $j \in\{1,2, \ldots, s\}$. Note that Algorithm 1 makes an orthogonal projection in each iteration. Hence, Algorithm 1 always selects an index from the support of $e$ in s-iterations. Then, Algorithm 1 will recover $\mathbf{x}^{*}$ from $\mathbf{b}=\mathbf{A} \mathbf{x}+\mathbf{e}^{*}$ in $s$ iterations.

From Theorem 3.5, we can see that the condition has no relationship with the original noise $\mathbf{e}^{*}$ and vector $\mathbf{x}^{*}$. They can be any length. In latter part, we will present some numerical examples to demonstrate this.

Theorem 3.6. Assume that $A$ is generated by the standard Gaussian random variable. If

$$
M>N \geq C \operatorname{slog}(M / s)
$$

for some constant $C>0$ and

$$
\frac{(\sqrt{M}-\sqrt{N}-10)^{2}}{\sqrt{M}+\sqrt{N}+10} \geq 2 \sqrt{N}
$$

Then, the condition (26) can be satisfied with high probability. 
Proof. Paper [21] shows that

$$
\beta_{s+1}\left(\mathbf{A}^{\top}\right)<\sqrt{N}(1+\delta)
$$

with high probability provided that $N \geq C \delta^{-2} s \log (M / s)$, where $C$ is some positive constant. Letting $\delta=1$, we can obtain that

$$
\sqrt{s+1} \beta_{s+1}\left(\mathbf{A}^{\top}\right)<2 \sqrt{N}
$$

with high probability provided that $N \geq C s \log (M / s)$. From [Corollary 35 ,[22]], Then for every $t \geq 0$, with probability at least $1-2 \exp \left(t^{2} / 2\right)$ one has

$$
\sqrt{M}-\sqrt{N}-t \leq \sigma_{\min }(\mathbf{A}) \leq\|\mathbf{A}\|_{2} \leq \sqrt{M}+\sqrt{N}+t .
$$

We choose $t=10$, then with high probability,

$$
\frac{\sigma_{\min }^{2}(\mathbf{A})}{\|\mathbf{A}\|_{2}} \geq \frac{(\sqrt{M}-\sqrt{N}-10)^{2}}{\sqrt{M}+\sqrt{N}+10} .
$$

With the deduction above, the condition (26) can be satisfied with high probability.

\section{Numerical results}

In this subsection, we provide the numerical results of the Algorithm 1 applied to robust regression. These results contain three parts: the first one focuses on the behavior of Algorithm 1; the other one compares Algorithm 1 with LAD; the third one applies Algorithm 1 to solving the TV- $L_{1}$ problem. In the experiments, the relative error is recorded as

$$
\text { RelErr }=\frac{\left\|\mathbf{x}^{k}-\mathbf{x}^{*}\right\|_{2}}{\left\|\mathbf{x}^{*}\right\|_{2}}
$$

The matrix $\mathbf{A} \in \mathbb{R}^{M \times N}$ and $\mathbf{x}^{*} \in \mathbb{R}^{M}$ are generated by Gaussian or Bernoulli random variable. The observed vector is generated as

$$
\mathbf{b}=\mathbf{A} \mathbf{x}^{*}+\mathbf{e}^{*}
$$

where $\mathbf{e}^{*} \in \mathbb{R}^{M}$ is the noise with $s$ nonzero elements. 


\subsection{Algorithm behavior}

In the first test, we choose $M=500, N=200$ and $s=50$. The nonzero elements of noise $\mathbf{e}^{*}$ are generated by standard Gaussian variable. For both Gaussian and Bernoulli cases, Figure 1 reports the estimated vector and the original one. The time costed are also presented. From the results, we can see that the greedy method is quite fast and valid.

In the second test, we still choose $M=500, N=200$ and $s=50$. But the nonzero elements of noise $\mathbf{e}^{*}$ are generated by $N\left(0, \sigma^{2}\right)$. We vary $\sigma$ from 0.01 to 100 , Figure 2 presents the relative errors. And we can see that all the relative errors are less than $10^{-3}$. As the length of the noise increases, the algorithm is more accurate.

\subsection{Comparison with other methods}

In this part, we compare the performance of Algorithm 1 and LAD, Sestimate, MM-estimate, LMS for the robust regression. We set $M=300$, $N=100$ and vary the sparsity $s$ from 0 to 150 . If the relative error RelErr $\leq 10^{-3}$, the recovery is viewed as a successful one. Figure 3 presents the reconstructing ability under different types of sampling matrices and vectors. From the results, we can see that Algorithm still works even $1 / 3$ of the observation is contaminated. And the greedy algorithm is more powerful than other four methods.

The second test is comparing the speed of the two algorithms. We set $M=500, N=200$. For different $s$, Table 1 presents the time costed by the algorithms. In the table, we use "GG" to express "Gaussian matrix Gaussian vector". The other symbols are similar. We see that the time costed by LAD is unstable; this is because, the LAD is constant of a linear program which may fall into a bad-calculated case. However, the greedy one performs much stabler; when $s$ is fixed, the time costed in different cases are almost similar. From the table, we can see that the greedy method is much faster and more accurate.

In the third test, we first consider the least square for a set of 2-dimensional points. In these points, 2 points are contaminated, and they contribute little to the data analysis. We use greedy method and other robust methods for the linear regression. We also present the result generated by Least Square regression (LS). Finally, we calculate the MSE without the two contaminated points. The MSE for Greedy, LAD, LMS, MM, S and LS are 2.3, 2.5, 3.8, 2.7, 2.8 and 5.2, respectively. The results in Figure 4 show that greedy method performs better. We also consider the multidimensional robust regression. 
Note that in applications, the parameters are always sparse. By this ideal and inspiration by the paper [25], we add a sparse item to the LAD and obtain the modified LAD (MLAD) as

$$
\min _{x}\|b-A x\|_{1}+\lambda\|x\|_{1} .
$$

Under different dimensions, Table 2 presents the MSE of different methods. The greedy method still works better.

\subsection{Application to sparse noise removal}

In this part, we apply Algorithm 1 to a 1-D sparse noise removal. We consider a vector $\mathbf{x}^{*} \in \mathbb{R}^{1024}$. And the noise is $\mathbf{e}^{*}$ which has $s$ nonzero elements. The observed vector is $b=\mathbf{x}^{*}+\mathbf{e}^{*}$. We choose $\mathbf{A}=[\lambda \mathbf{T}, \mathbf{I d}]^{\top}$ and $\mathbf{b}=[\mathbf{0}, \mathbf{d}]$, and use the greedy algorithm to obtain $\overline{\mathbf{x}}$ such that $\mathbf{b}-\mathbf{A} \overline{\mathbf{x}}$ is sparse.

\section{Conclusion}

In this paper, we propose a greedy method for the robust linear regression. Such an algorithm performs quite faster than existing method. We also prove that the greed algorithm can estimate the vector well if the noise is sparse and the sampling matrix satisfies certain property. The numerical results demonstrate the efficiency of the algorithm.

\section{Acknowledgments}

The authors are really indebted to the editors and anonymous referees for their useful suggestions and help to improve the quality of the manuscript. We are grateful for the National Science Foundation of China (No.61402495).

[1] Seber G A F, Lee A J. Linear regression analysis[M]. John Wiley \& Sons, 2012 .

[2] Montgomery D C, Peck E A, Vining G G. Introduction to linear regression analysis[M]. John Wiley \& Sons, 2012.

[3] Rousseeuw P J, Leroy A M. Robust regression and outlier detection[M]. John Wiley \& Sons, 2005.

[4] Yu C, Yao W, Bai X. Robust Linear Regression: A Review and Comparison[J]. arXiv preprint arXiv:1404.6274, 2014. 
[5] Huber P J. Robust statistics[M]. Springer Berlin Heidelberg, 2011.

[6] Ying Z, Jung S H, Wei L J. Survival analysis with median regression models[J]. Journal of the American Statistical Association, 1995, 90(429): 178-184.

[7] Bloomfield P, Steiger W. Least absolute deviations: Theory, applications and algorithms[M]. Springer Science \& Business Media, 2012.

[8] Schöbel A. Anchored hyperplane location problems[J]. Discrete and Computational Geometry, 2003, 29(2): 229-238.

[9] Brooks J P, Dulá J H. The L1-norm best-fit hyperplane problem[J]. Applied mathematics letters, 2013, 26(1): 51-55.

[10] Clarkson K L. Subgradient and sampling algorithms for 11 regression[C]//Proceedings of the sixteenth annual ACM-SIAM symposium on Discrete algorithms. Society for Industrial and Applied Mathematics, 2005: 257266.

[11] Dasgupta A, Drineas P, Harb B, et al. Sampling algorithms and coresets for $\ell_{p}$ regression[J]. SIAM Journal on Computing, 2009, 38(5): 2060-2078.

[12] Clarkson K L, Drineas P, Magdon-Ismail M, et al. The Fast Cauchy Transfor$\mathrm{m}$ and faster robust linear regression $[\mathrm{C}] / /$ Proceedings of the Twenty-Fourth Annual ACM-SIAM Symposium on Discrete Algorithms. SIAM, 2013: 466477.

[13] Yang J, Zhang Y, Yin W. An efficient TVL1 algorithm for deblurring multichannel images corrupted by impulsive noise[J]. SIAM Journal on Scientific Computing, 2009, 31(4): 2842-2865.

[14] Rudin L I, Osher S, Fatemi E. Nonlinear total variation based noise removal algorithms[J]. Physica D: Nonlinear Phenomena, 1992, 60(1): 259-268.

[15] Tropp J, Gilbert A C. Signal recovery from random measurements via orthogonal matching pursuit[J]. Information Theory, IEEE Transactions on, 2007, 53(12): 4655-4666.

[16] Mo Q, Shen Y. A remark on the restricted isometry property in orthogonal matching pursuit $[\mathrm{J}]$. Information Theory, IEEE Transactions on, 2012, 58(6): 3654-3656.

[17] Mo Q. A Sharp Restricted Isometry Constant Bound of Orthogonal Matching Pursuit[J]. arXiv preprint arXiv:1501.01708, 2015. 
[18] Foucart S, Rauhut H. A mathematical introduction to compressive sensing[M]. Basel: Birkhäuser, 2013.

[19] Demmel J W. Applied numerical linear algebra[M]. SIAM, 1997.

[20] Candes E J, Tao T. Decoding by linear programming[J]. Information Theory, IEEE Transactions on, 2005, 51(12): 4203-4215.

[21] Baraniuk R, Davenport M, DeVore R, et al. A simple proof of the restricted isometry property for random matrices $[\mathrm{J}]$. Constructive Approximation, 2008, 28(3): 253-263.

[22] Rudelson M, Vershynin R. Non-asymptotic theory of random matrices: extreme singular values[C]//PROCEEDINGS OF THE INTERNATIONAL CONGRESS OF MATHEMATICIANS. 2010.

[23] Yang J, Zhang Y, Yin W. A fast alternating direction method for TVL1-L2 signal reconstruction from partial Fourier data[J]. Selected Topics in Signal Processing, IEEE Journal of, 2010, 4(2): 288-297.

[24] Rousseeuw P J. Least median of squares regression[J]. Journal of the American statistical association, 1984, 79(388): 871-880.

[25] Liu H, Liu Y, Sun F. Robust exemplar extraction using structured sparse coding $[\mathrm{J}]$. IEEE transactions on neural networks and learning systems, 2015, 26(8): 1816-1821.

[26] Yohai V J. High breakdown-point and high efficiency robust estimates for regression[J]. The Annals of Statistics, 1987: 642-656.

[27] Rousseeuw P, Yohai V. Robust regression by means of Sestimators $[\mathrm{M}] / /$ Robust and nonlinear time series analysis. Springer US, 1984: 256-272. 


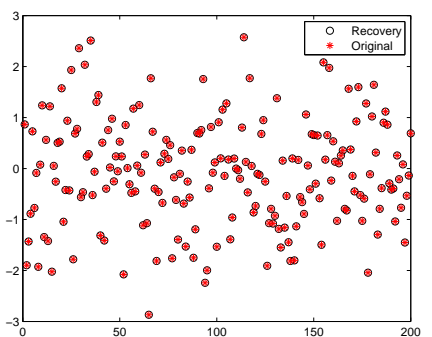

(a) Gaussian matrix Gaussian (b) Gaussian matrix Bernoulli vector $\operatorname{RelErr}=2.6 \times 10^{-4} 3.1 \mathrm{~s}$

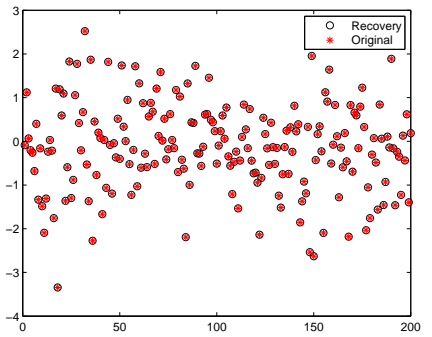

(c) Bernoulli matrix Gaussian vec- (d) Bernoulli matrix Bernoulli tor RelErr $=5.3 \times 10^{-5} 2.7 \mathrm{~s}$

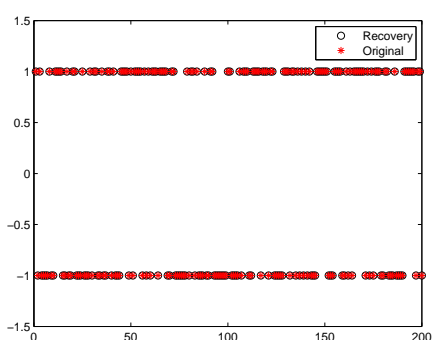

vector $\operatorname{RelErr}=1.6 \times 10^{-4} 2.9 \mathrm{~s}$

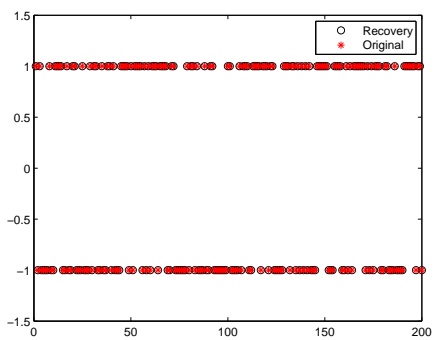

vector RelErr $=1.5 \times 10^{-4} 3.2 \mathrm{~s}$

Figure 1: Reconstructing results and time costed 

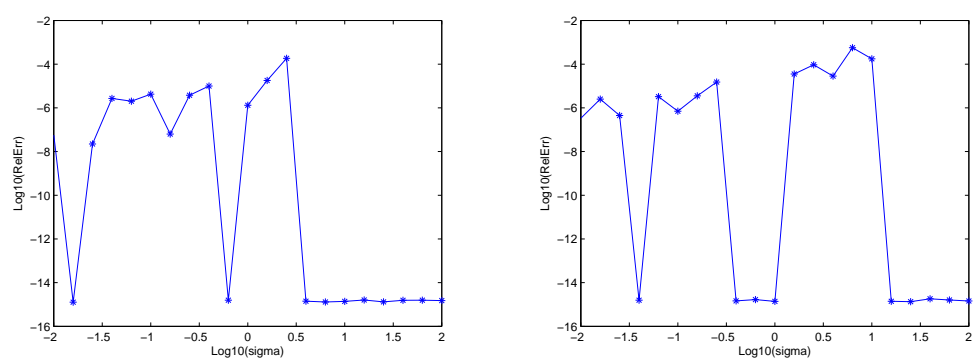

(a) Gaussian matrix Gaussian (b) Gaussian matrix Bernoulli vector vector
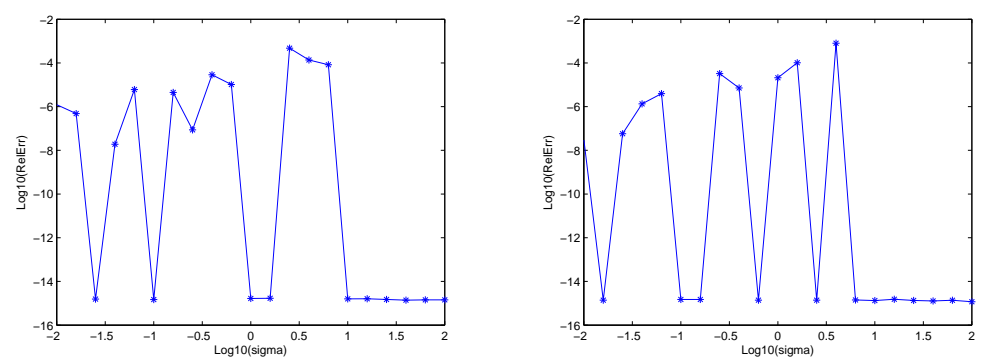

(c) Bernoulli matrix Gaussian vec- (d) Bernoulli matrix Bernoulli tor vector

Figure 2: Relative errors versus the length of the noise 

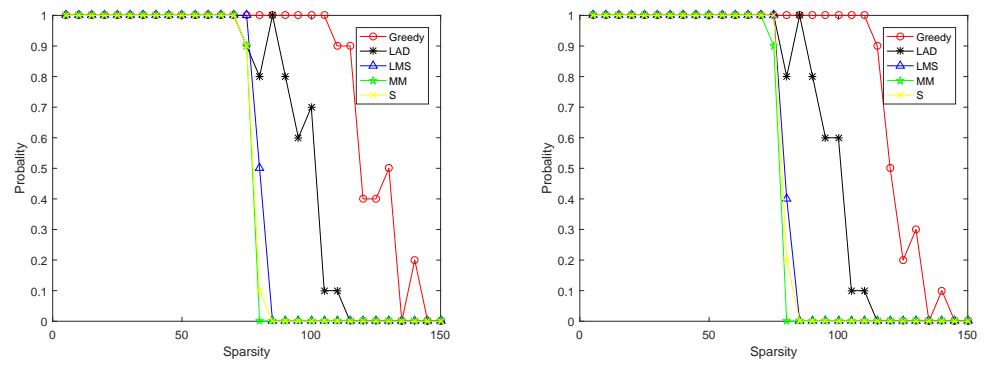

(a) Gaussian matrix Gaussian (b) Gaussian matrix Bernoulli vector vector
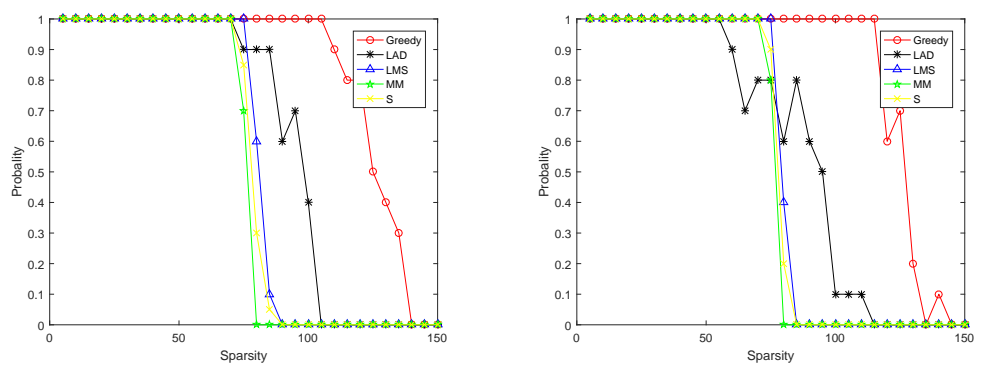

(c) Bernoulli matrix Gaussian vec- (d) Bernoulli matrix Bernoulli tor vector

Figure 3: Reconstructing ability versus the sparsity

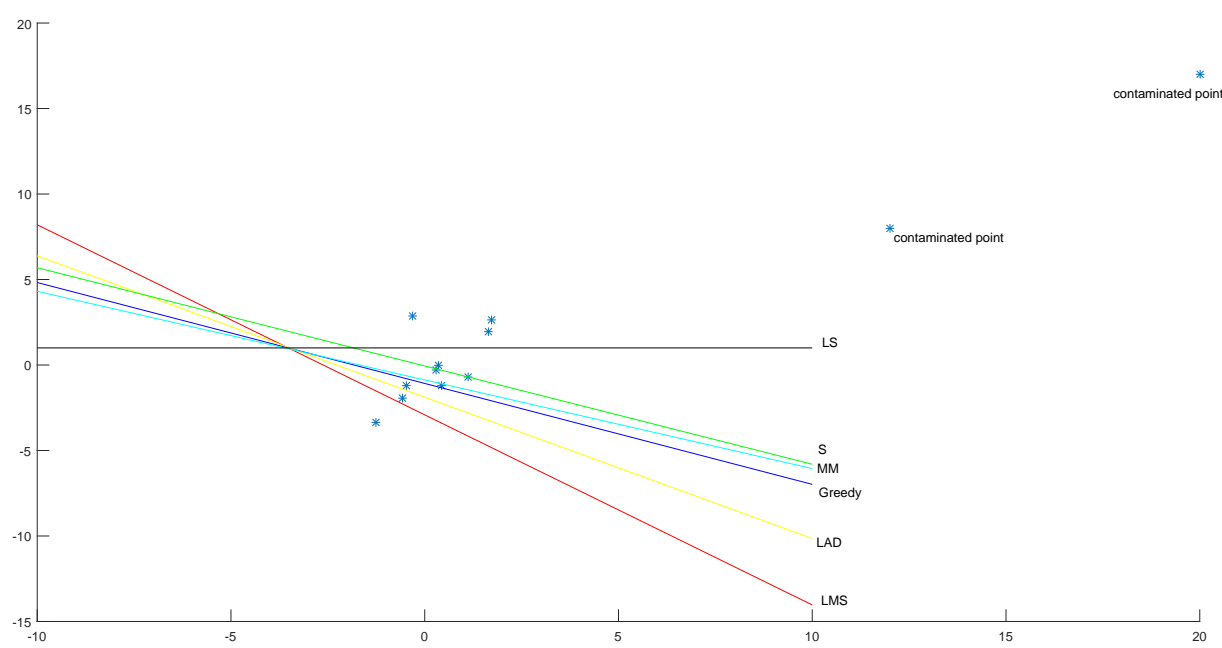

Figure 4: 2-dimensional regression 
Table 1: Relative error and time costed for different matrices

\begin{tabular}{|c|c|c|c|c|c|c|c|c|c|c|c|}
\hline \multicolumn{2}{|c|}{ scenario } & \multicolumn{2}{|c|}{ Greedy method } & \multicolumn{2}{|c|}{ LAD } & \multicolumn{2}{|c|}{ LMS } & \multicolumn{2}{|c|}{ MM } & \multicolumn{2}{|c|}{$\mathrm{S}$} \\
\hline & & RelErr & Time & RelErr & Time & RelErr & Time & RelErr & Time & RelErr & Time \\
\hline \multirow{4}{*}{$\mathrm{s}=20$} & GG & $1.2 \mathrm{e}-15$ & $0.4 \mathrm{~s}$ & $5.1 \mathrm{e}-10$ & $9.2 \mathrm{~s}$ & $1.8 \mathrm{e}-15$ & $13.2 \mathrm{~s}$ & $1.5 \mathrm{e}-15$ & $0.9 \mathrm{~s}$ & $2.3 \mathrm{e}-15$ & $10.3 \mathrm{~s}$ \\
\hline & GB & $1.3 \mathrm{e}-15$ & $0.5 \mathrm{~s}$ & $6.3 \mathrm{e}-10$ & $5.6 \mathrm{~s}$ & $2.1 \mathrm{e}-15$ & $14 \mathrm{~s}$ & $4.2 \mathrm{e}-13$ & $0.9 \mathrm{~s}$ & $8.2 \mathrm{e}-15$ & $7.3 \mathrm{~s}$ \\
\hline & $\mathrm{BG}$ & $1.4 \mathrm{e}-15$ & $0.5 \mathrm{~s}$ & $1.5 \mathrm{e}-8$ & $14.3 \mathrm{~s}$ & $1.5 \mathrm{e}-15$ & $14.9 \mathrm{~s}$ & $1.2 \mathrm{e}-15$ & $0.9 \mathrm{~s}$ & $3.1 \mathrm{e}-15$ & $8.9 \mathrm{~s}$ \\
\hline & $\mathrm{BB}$ & $1.5 \mathrm{e}-15$ & $0.4 \mathrm{~s}$ & $9.0 \mathrm{e}-10$ & $5.4 \mathrm{~s}$ & $1.6 \mathrm{e}-15$ & 13.7 & 1.e-15 & $0.8 \mathrm{~s}$ & $4.1 \mathrm{e}-15$ & $7.9 \mathrm{~s}$ \\
\hline \multirow{4}{*}{$\mathrm{s}=40$} & $\mathrm{GG}$ & $1.6 \mathrm{e}-15$ & $0.7 \mathrm{~s}$ & $9.1 \mathrm{e}-9$ & $10.6 \mathrm{~s}$ & $1.6 \mathrm{e}-15$ & $14.3 \mathrm{~s}$ & $2.1 \mathrm{e}-15$ & $0.9 \mathrm{~s}$ & $4.4 \mathrm{e}-15$ & $9.2 \mathrm{~s}$ \\
\hline & GB & $1.2 \mathrm{e}-15$ & $0.6 \mathrm{~s}$ & $8.2 \mathrm{e}-9$ & $7.4 \mathrm{~s}$ & $4.6 \mathrm{e}-15$ & $12.2 \mathrm{~s}$ & $5.2 \mathrm{e}-15$ & $0.9 \mathrm{~s}$ & $6.5 \mathrm{e}-15$ & $8.9 \mathrm{~s}$ \\
\hline & $\mathrm{BG}$ & $1.5 \mathrm{e}-15$ & $0.7 \mathrm{~s}$ & $3.0 \mathrm{e}-9$ & $7.2 \mathrm{~s}$ & $2.4 \mathrm{e}-14$ & $15.1 \mathrm{~s}$ & $7.2 \mathrm{e}-15$ & $1.0 \mathrm{~s}$ & $2.7 \mathrm{e}-15$ & $8.7 \mathrm{~s}$ \\
\hline & $\mathrm{BB}$ & $1.5 \mathrm{e}-15$ & $0.7 \mathrm{~s}$ & $6.0 \mathrm{e}-9$ & $16.7 \mathrm{~s}$ & $2.8 \mathrm{e}-15$ & $14.8 \mathrm{~s}$ & $1.1 \mathrm{e}-15$ & $0.9 \mathrm{~s}$ & $4.2 \mathrm{e}-15$ & $10.1 \mathrm{~s}$ \\
\hline \multirow{4}{*}{$\mathrm{s}=60$} & GG & $1.4 \mathrm{e}-15$ & $1.0 \mathrm{~s}$ & $3.1 \mathrm{e}-9$ & $6.2 \mathrm{~s}$ & $2.2 \mathrm{e}-15$ & $12.4 \mathrm{~s}$ & $1.7 \mathrm{e}-15$ & $1.3 \mathrm{~s}$ & $4.4 \mathrm{e}-15$ & $9.4 \mathrm{~s}$ \\
\hline & GB & $1.3 \mathrm{e}-15$ & $1.1 \mathrm{~s}$ & $5.9 \mathrm{e}-9$ & $8.7 \mathrm{~s}$ & $8.4 \mathrm{e}-15$ & $12.5 \mathrm{~s}$ & $1.2 \mathrm{e}-15$ & $1.2 \mathrm{~s}$ & $6.1 \mathrm{e}-15$ & $9.8 \mathrm{~s}$ \\
\hline & $\mathrm{BG}$ & $1.6 \mathrm{e}-15$ & $1.0 \mathrm{~s}$ & $2.4 \mathrm{e}-9$ & $10.2 \mathrm{~s}$ & $1.4 \mathrm{e}-15$ & 13.1s & $4.3 \mathrm{e}-15$ & $1.2 \mathrm{~s}$ & $2.1 \mathrm{e}-15$ & $9.6 \mathrm{~s}$ \\
\hline & $\mathrm{BB}$ & $1.4 \mathrm{e}-15$ & $1.0 \mathrm{~s}$ & $6.7 \mathrm{e}-9$ & $7.6 \mathrm{~s}$ & $2.5 \mathrm{e}-15$ & $12.7 \mathrm{~s}$ & $2.8 \mathrm{e}-15$ & $1.3 \mathrm{~s}$ & $1.2 \mathrm{e}-15$ & $10.2 \mathrm{~s}$ \\
\hline \multirow{4}{*}{$\mathrm{s}=80$} & GG & $1.3 \mathrm{e}-15$ & $1.2 \mathrm{~s}$ & $3.6 \mathrm{e}-9$ & $11.9 \mathrm{~s}$ & $1.8 \mathrm{e}-15$ & $12.4 \mathrm{~s}$ & $1.6 \mathrm{e}-15$ & $1.4 \mathrm{~s}$ & $1.5 \mathrm{e}-15$ & $12.6 \mathrm{~s}$ \\
\hline & GB & $1.4 \mathrm{e}-15$ & $1.3 \mathrm{~s}$ & $8.1 \mathrm{e}-9$ & $6.8 \mathrm{~s}$ & $1.4 \mathrm{e}-15$ & $12.2 \mathrm{~s}$ & $1.6 \mathrm{e}-15$ & $1.3 \mathrm{~s}$ & $2.1 \mathrm{e}-15$ & $13.0 \mathrm{~s}$ \\
\hline & $\mathrm{BG}$ & $1.7 \mathrm{e}-15$ & $1.3 \mathrm{~s}$ & $4.7 \mathrm{e}-9$ & $10.7 \mathrm{~s}$ & $2.4 \mathrm{e}-15$ & $11.8 \mathrm{~s}$ & $1.1 \mathrm{e}-15$ & $1.5 \mathrm{~s}$ & $4.2 \mathrm{e}-15$ & $12.8 \mathrm{~s}$ \\
\hline & $\mathrm{BB}$ & $1.4 \mathrm{e}-15$ & $1.2 \mathrm{~s}$ & $1.7 \mathrm{e}-9$ & $8.2 \mathrm{~s}$ & $3.1 \mathrm{e}-15$ & $12.9 \mathrm{~s}$ & $1.3 \mathrm{e}-15$ & $1.5 \mathrm{~s}$ & $2.7 \mathrm{e}-15$ & $12.7 \mathrm{~s}$ \\
\hline \multirow{4}{*}{$s=100$} & GG & $1.4 \mathrm{e}-15$ & $1.3 \mathrm{~s}$ & $9.8 \mathrm{e}-9$ & $6.6 \mathrm{~s}$ & $1.8 \mathrm{e}-15$ & $12.6 \mathrm{~s}$ & $3.5 \mathrm{e}-7$ & $2.1 \mathrm{~s}$ & $8.2 \mathrm{e}-7$ & $11.6 \mathrm{~s}$ \\
\hline & GB & $1.6 \mathrm{e}-15$ & $1.4 \mathrm{~s}$ & $1.0 \mathrm{e}-9$ & $4.7 \mathrm{~s}$ & $1.4 \mathrm{e}-15$ & $12.2 \mathrm{~s}$ & $6.2 \mathrm{e}-7$ & $2.1 \mathrm{~s}$ & $2.3 \mathrm{e}-7$ & $12.2 \mathrm{~s}$ \\
\hline & $\mathrm{BG}$ & $1.5 \mathrm{e}-15$ & $1.5 \mathrm{~s}$ & $1.2 \mathrm{e}-6$ & $26.3 \mathrm{~s}$ & $3.1 \mathrm{e}-15$ & $12.4 \mathrm{~s}$ & $1.2 \mathrm{e}-7$ & $2.5 \mathrm{~s}$ & $4.1 \mathrm{e}-7$ & $12.1 \mathrm{~s}$ \\
\hline & $\mathrm{BB}$ & $1.4 \mathrm{e}-15$ & $1.4 \mathrm{~s}$ & $8.3 \mathrm{e}-9$ & $5.8 \mathrm{~s}$ & $2.3 \mathrm{e}-15$ & $12.3 \mathrm{~s}$ & $2.5 \mathrm{e}-7$ & $2.4 \mathrm{~s}$ & $3.1 \mathrm{e}-7$ & $11.3 \mathrm{~s}$ \\
\hline
\end{tabular}

Table 2: MSE of different dimensional test data by different methods

\begin{tabular}{ccccccc}
\hline scenario & Greedy method & LAD & M-LAD & LMS & MM & S \\
\hline $100 \times 50$ & 1.7 & 2.4 & 2.1 & 3.5 & 2.7 & 2.8 \\
$150 \times 70$ & 1.6 & 2.2 & 2.0 & 3.6 & 2.7 & 2.7 \\
$200 \times 100$ & 1.7 & 2.3 & 2.2 & 3.6 & 2.8 & 2.7 \\
$250 \times 120$ & 1.7 & 2.3 & 2.2 & 3.6 & 2.7 & 2.7 \\
\hline
\end{tabular}



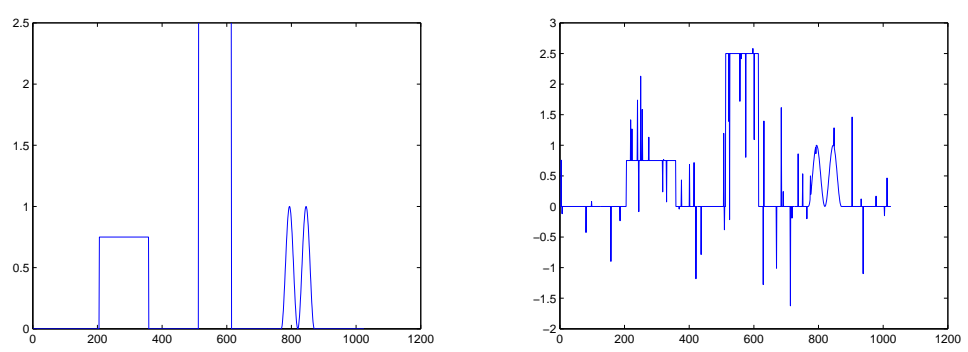

(a) Gaussian matrix Gaussian (b) Gaussian matrix Bernoulli vector vector

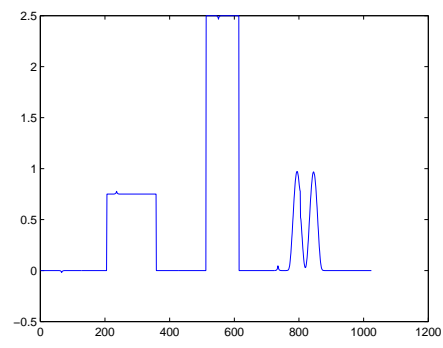

(c) $33.2 \mathrm{~s}$ ReErr $=0.0101$

Figure 5: Reconstructing ability versus the sparsity 